\title{
SCD - Stem Cell Differentiation Toward Osteoblast Onboard the International Space Station
}

\author{
Silvia Bradamante ${ }^{1,2} \cdot$ Damariz Rivero $^{1} \cdot$ Livia Barenghi $^{1,3} \cdot$ Michele Balsamo $^{4} \cdot$ Simone Paolo Minardi ${ }^{5}$. \\ Francesco Vitali $^{6}$. Duccio Cavalieri ${ }^{6}$
}

Received: 5 February 2018 / Accepted: 28 August 2018 / Published online: 19 September 2018

(C) The Author(s) 2018

\begin{abstract}
Microgravity produces a variety of physical, chemical, and biological cues leading to an intricate and largely unresolved network of mechanosensitive molecules, transduction pathways, oxidative stress-related responses, and adaptations. The bone loss observed in astronauts and animal models after spaceflight is attributable to alterations in the bone tissue formation that depends on the continuous remodelling through the activities of bone-resorbing osteoclasts of hematopoietic lineage and bone-forming osteoblast of mesenchymal origin. Focusing on osteogenic differentiation, we present the results of the "SCD - Stem Cells Differentiation" experiment, aiming to determine how human bone marrow stem cells (hBMSCs) react to a prolonged (approx. 2 weeks) exposure to microgravity in terms of growth, and differentiation when treated with a physiological osteo-inducer as 1,25-dihydroxy vitamin D (Vit D3). The experiment was selected by the European Space Agency and transferred to ISS with the Soyuz-TMA- 16M (ISS 42S). It was carefully prepared because experiments performed on ISS remain a uniquely exceptional means of clarifying the microgravitational effects on osteogenesis, often only partially activated and detectable under simulated conditions. Because of the substantial reduction in calcification observed (about 50\% inflight vs. on-ground control), we looked at significantly affected pathways in hBMSCs grown in microgravity vs. on-ground controls. Genome-wide expression changes were assessed via microarray and next generation sequencing (NGS) and integrated with exosomal mi-RNA measurements. Multi-scale pathway analysis of the omics datasets revealed evidence of cell cycle arrest, occurring with a number of osteogenic gene markers, but without indications of adipogenesis, senescence and/or apoptosis.
\end{abstract}

Keywords Microgravity $\cdot$ Epigenetics $\cdot$ Transcriptomics $\cdot$ Bone mass loss · Aging

This article belongs to the Topical Collection: Interdisciplinary Science Challenges for Gravity Dependent Phenomena in Physical and Biological Systems

Guest Editors: Jens Hauslage, Ruth Hemmersbach, Valentina Shevtsova

Electronic supplementary material The online version of this article (https://doi.org/10.1007/s12217-018-9653-2) contains supplementary material, which is available to authorized users.

Silvia Bradamante

silvia.bradamante@gmail.com

1 Institute of Molecular Science and Technologies, CNR, via Golgi, 19, 20133 Milan, Italy

2 NMR Lab, University of Milano-Bicocca, viale dell'Innovazione, 10, I-20126 Milano, Italy

3 Integrated Orthodontic Services srl, Via Cavour 52 C, 23900 Lecco, Italy

\section{Introduction}

Mesenchymal stem cells (MSCs) are multipotent cells that are capable of differentiating into mature cells of several mesenchymal tissues, such as fat and bone, depending on the contribution made by a variety of external chemical, physical, and biological cues (Kokabu et al. 2016; Chen et al. 2016a). Indeed, MSCs respond by activating mechanosensors

4 Kayser Italia S.r.l., Via di Popogna, 501, 57128 Livorno, Italy

5 Cogentech (Consortium for Genomic Technologies), c/o IFOM-IEO Campus, Via Adamello, 16 - 20139 Milan, Italy

6 Department of Biology, University of Florence, Via Madonna del Piano 6, 50019 Sesto Fiorentino, Firenze, Italy 
that mediate the process of biological mechanotransduction. Nevertheless, according to many authors, the scientific progresses in understanding the mechanisms of adipogenic / osteogenic differentiation of MSCs on ground still need confirmation (Chen and Jacobs 2013; Ingber et al. 2014; Huang et al. 2015).

Compared to on-ground conditions, microgravity $(\mu g)$ produces a different and complex altered physical environment of forces (adhesion, tension, pressure, etc.) (Louis et al. 2015; Arfat et al. 2014; Vorselen et al. 2014; Bradamante et al. 2014; Versari et al. 2016) and leads to an intricate network on mechanosensitive molecules, transduction pathways, oxidative stress-related and adaptive responses. Nevertheless again, today we still do not have a clear answer to the bone-loss problem (Nagaraja and Risin 2013; Vico et al. 2017) that occurs in a site-specific and compartment-specific manner not only during, but also after spaceflight.

During long-term missions, astronauts face many physiological challenges. Living in the low-gravitational environment implies a redistribution of body fluids and unloading of weight-bearing bones and postural muscles (Vico et al. 2000; Adams et al. 2003) with consequences such as motion sickness, cardiovascular deconditioning, muscle atrophy, and a loss of bone mass (Grimm et al. 2016). Many of these diseases parallel the aging pathologies, but they occur and develop much more rapidly in space (Vernikos and Schneider 2010).

Bone loss has been observed in astronauts and animals after spaceflight and in on-ground simulated microgravity $(\operatorname{sim}-\mu g)$ experiments, from human bed rest, animal hindlimb unloading experiments, to in vitro studies of cellular models (Nagaraja and Risin 2013; Vernikos and Schneider 2010; van Deursen 2014). Despite the numerous reports, the pathology remains a key concern and development of effective countermeasures is still a major task.

In the past, researchers considered and investigated the failures in osteoblast and osteoclast activity responsible for bone mass loss in $\mu \mathrm{g}$ (Grimm et al. 2016). Here, the focus is on stem cells (SCs), which play a major role in the maintenance of bone mass, being the main source of osteoblasts during bone remodelling and repair. The recruitment of an adequate number of osteoblasts is dependent on the availability of human Mesenchymal Stem Cells (hMSCs) and their proper response to growth, differentiation, and chemotactic signals in the microenvironment (Lian et al. 2012). It is noteworthy that today there is increasing evidence that most of the diseases related to aging, including osteoporosis, are associated with a progressive decline in the number and/or function of SCs (van Deursen 2014).

Our paper describes the results of the "SCD - Stem Cells Differentiation" experiment, selected by ESA via ILSRA2009 (International Research Announcement for Research in Space Life Sciences at the International Space Station) and launched to ISS on March 27, 2015 with the SoyuzTMA-16M (ISS 42S) mission. The aim of this study is to determine how hBMSCs react to a prolonged (approx. 2 weeks) exposure to $\mu \mathrm{g}$ in terms of growth, senescence and osteogenic differentiation, when treated with 1,25dihydroxy vitamin D3 (Vit D3) (Bradamante et al. 2014). The experiment was carefully prepared (Versari et al. 2016) since experiments performed on the International Space Station (ISS) remain an exceptional tool for clarifying the effects of $\mu \mathrm{g}$ on stem cells and their differentiation. Indeed due to the limits of the $\mu \mathrm{g}$ simulators (Bradamante et al. 2014; Luna et al. 2015; Yan et al. 2015) the results of experiments conducted in sim- $\mu \mathrm{g}$ (Zayzafoon et al. 2004), although instructive, can be very much affected by the simulator design.

We first looked at significantly affected pathways in hBMSCs grown in flight and on ground $(\mu g v s .1 g)$, and then compared the differentiation program promoted by Vit D3 in flight and on ground ( $\mu g v s .1 g$, resembling the "in vivo" situation). Using selected genomic approaches, ranging from microarray to Next Generation Sequencing (NGS) analyses, we show how $\mu g$ stresses hBMSCs involving important biological pathways with selected osteogenic and senescence gene markers, but without indications of adipogenesis and apoptosis. Our data point to major rearrangements of extracellular matrix stiffness in osteogenic conditions. In addition, the observed hyperproduction of microvesicles/exosomes is consistent with the miRNAs found in spent media.

\section{Materials and Methods}

\section{Cell Culture}

Human bone marrow MSCs (hBMSCs) were kindly provided by Ospedale Sacco, University of Milan Italy, with the collaboration of prof. JAM Maier and her group, dr. Sara Castiglioni and dr. Cazzaniga et al. (2016). We thank all of them for their dedication in the preparation of the SCD experiment that was extensively tested and described before the spaceflight mission (Versari et al. 2016).

Briefly, human BMSCs cells were cultured on Thermanox collagen coated coverslips for $\sim 17$ days either in Standard Medium (SM) or in Osteogenic Medium (OM), the composition of which were the following: a) SM: DMEM (Dulbecco's Modified Eagle's medium) supplemented with $12.5 \mathrm{mM}$ HEPES, $10 \mathrm{U} / \mathrm{mL}$ heparin, $200 \mathrm{mM}$ glutamine, $500 \mu \mathrm{g} / \mathrm{mL}$ streptomycin sulphate, $600 \mu \mathrm{g} / \mathrm{mL}$ penicillin; b) OM: SM added with $0.1 \mathrm{M}$ ascorbic acid, 10 $\mathrm{mM} \beta$-glycerophosphate, and $10^{-8} \mathrm{M} 1,25(\mathrm{OH})_{2} \mathrm{D} 3$ (Vit D3). All chemicals were purchased from Sigma Aldrich, 
Saint Louis, MO, USA. Thermanox coverslips were purchased from Thermo Fisher Scientific Inc.Waltham, MA USA. Due to safety constraint in space, we selected NOTOXhisto (Scientific Device Laboratory, USA) as nontoxic fixative (Versari et al. 2016).

\section{SCD Experiment}

The SCD flight set consisted of 24 experiment hardware (EH) modules, 12 flight modules (Fm) and 12 ground modules (Gm), developed by Kayser Italia (Livorno, Italy; http:// www.kayser.it/, each of which had one experiment unit (EU) integrated into the KUBIC interface container single level (KIC-SL). Each EU (Fig. 1) consisted of a brick made of biologically compatible plastic [polyetheretherketone (PEEK)] containing 5 cylinders (for the medium and chemicals), a cell culture chamber (CC), and connecting channels. Five small valves were placed to separate the different fluids and the CC. Each cylinder had a piston to inject a new fluid into the CC; the waste medium was collected in the previously emptied cylinder and suitably preserved. The SCD flight set was identical to that used for the successful SPHINX experiment (Versari et al. 2013b). During the first 3 days in space the temperature in the Soyuz vehicle slowly decreased: $27^{\circ} \mathrm{C}$ was the temperature recorded for the samples before docking and installation in the KUBIK incubator on board the ISS. Before the flight, in our laboratory, we verified that this temperature would not jeopardize the success of the experiment by culturing hBMSCs in the $\mathrm{EH}$ modules at $27^{\circ} \mathrm{C}$ for selected time intervals and determining that they were vital (data not shown).

It is known that cell shape and mechanical regulation (McBeath et al. 2004; Eyckmans et al. 2012) drives hMSC commitment. Therefore, in order to optimize proliferation and differentiation during the 14 days spaceflight experiment, the cells were seeded at a density of 25000 cells $/ \mathrm{cm}^{2}$ to reach an estimated $80 \%$ confluence 3 days after the launch $(\mathrm{L}+3 \mathrm{~d})$ before the first medium exchange. Figure 2 shows some essential parts of the spaceflight mission. Briefly, 24 Experimental Units (EUs) were prepared as indi-

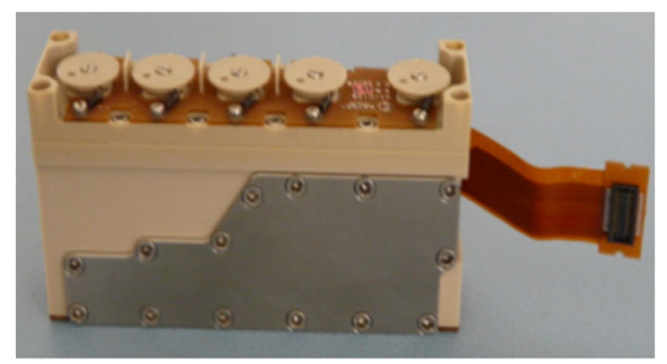

Fig. 1 The SCD EU with integrated electronics cated in Fig. 2 (panel a); 12 electronically integrated EUs inserted in the (KIC-SL) to form the experimental hardware $(\mathrm{EH})$ modules, were transferred in soft pouches by the Soyuz-TMA-16M (ISS 42S, launched on March 27, 2015) to the ISS.

The experiment profile is that detailed in Fig. 2 (panel b). Once on ISS we applied the following schedule:

1. Time-point $\mathbf{T}_{\mathbf{0}}$ : star symbol in Fig. 2. Installation of the EH in the KUBIK, registered temperature was $27^{\circ} \mathrm{C}$.

2. Time-point $\mathbf{T}_{\mathbf{0}}+\mathbf{7}$ hrs: step $\mathrm{C}$ in Fig. 2. Starting point of the experiment and first medium exchange (medium $\mathrm{SM}$ or $\mathrm{OM}$ ). From the previous time-point, temperature is raised to $36.5^{\circ} \mathrm{C}$ and will be maintained stable until the end of the experiment.

3. Time-point $\mathbf{T}_{\mathbf{0}}+\mathbf{7}$ days: step $\mathrm{B}$ in Fig. 2; second medium exchange (medium SM or OM).

4. Time-point $\mathbf{T}_{\mathbf{0}}+\mathbf{1 4}$ days: step $\mathrm{C}$ in Fig. 2; third medium exchange (PBS). The experiment was then stopped by means of two subsequent exchanges with RNAlater in eight EH modules and two subsequent exchanges with NOTOXhisto in the remaining four $\mathrm{EH}$

a

\begin{tabular}{|c|c|c|c|c|}
\hline \multicolumn{5}{|c|}{ hBMSC samples } \\
\hline \multirow{4}{*}{$\mu \mathrm{g}$} & Name & $\mathrm{n}$ & Medium & Fixative \\
\hline \multirow{4}{*}{$1 \mathrm{~g}$} & $\mathrm{Fm}-\mathrm{SM}$ & 4 & $\mathrm{SM}$ & RNAlater \\
\cline { 2 - 5 } & $\mathrm{Fm}-\mathrm{OM}$ & 4 & $\mathrm{OM}$ & RNAlater \\
\cline { 2 - 5 } & $\mathrm{Fm}-\mathrm{SM}$ & 2 & $\mathrm{SM}$ & NOTOXhisto \\
\cline { 2 - 5 } & $\mathrm{Fm}-\mathrm{OM}$ & 2 & $\mathrm{OM}$ & NOTOXhisto \\
\hline \multirow{4}{*}{} & $\mathrm{Gm}-\mathrm{SM}$ & 4 & $\mathrm{SM}$ & RNAlater \\
\cline { 2 - 5 } & $\mathrm{Gm}-\mathrm{OM}$ & 4 & $\mathrm{OM}$ & RNAlater \\
\cline { 2 - 5 } & $\mathrm{Gm}-\mathrm{SM}$ & 2 & $\mathrm{SM}$ & NOTOXhisto \\
\cline { 2 - 5 } & $\mathrm{GM}-\mathrm{OM}$ & 2 & $\mathrm{OM}$ & NOTOXhisto \\
\hline
\end{tabular}

SCD experiment profile

b

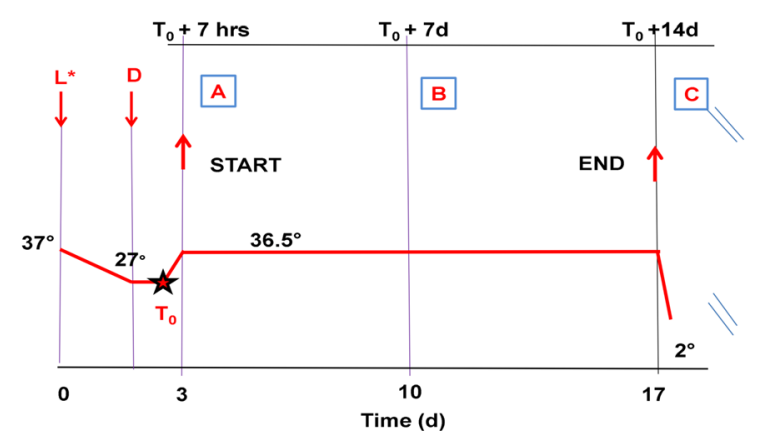

Fig. 2 SCD experimental planning and profile. (a) List of how 24 EUs of human BMSCs were cultured in different media and the used fixatives. $\mu \mathrm{g}$ : microgravity, g: gravity, Fm: flight model, $\mathrm{Gm}$ : ground model, SM: standard medium, OM: osteogenic medium, $\mathrm{N}$ : number of EUs. (b) The scheme describes the evolution of temperature with time and the main activities from the launch $\left(\mathrm{L}^{*}\right)$ of the SOYUZ followed by $8 \mathrm{~min}$ at $3.5 \mathrm{~g}$ to $\mathrm{A}: 1^{\text {st }}$ medium exchange, $\mathrm{B}: 2^{\text {nd }}$ medium exchange and the end of the SCD experiment with $\mathrm{C}: 3^{\text {rd }}$ medium exchange and fixation. Then the temperature in MELFI fridge was set at $2{ }^{\circ} \mathrm{C}$ until the return of the experiment on Earth 
modules; the fixative exchanges were separated by a 6 min interval. After each exchange, a protease inhibitor cocktail was added to the exhausted culture medium.

The EH modules were subsequently kept at $2{ }^{\circ} \mathrm{C}$ inside the MELFI fridge where they remained for longer than expected due to a delay ( $\sim 1$ month) in the return of the Soyuz to Earth. Indeed Soyuz -TMA-15M with Expedition 43 crew landed in Kazakhstan on June 11, 2015 and the $\mathrm{EH}$ modules were delivered to PI in Milan 2 days later. The other $12 \mathrm{EH}$ modules prepared in Baikonur were run in parallel using the same experimental protocol. All of the medium exchanges and fixation operations were automated based on the predefined timeline.

Within 2 days from the delivery to Milan all of the 24 EH modules were disassembled at Kayser Italia in Livorno, Italy.

Cell samples were in good condition. In conclusion, all of the SCD EH modules (in flight and on Earth) were correctly activated, and cells and culture media have been fixed in accordance with the timeline. In the end, each module provided a $230-\mathrm{mm}^{2}$ cell sample and the related preserved media (media A - C, $1.8 \mathrm{ml}$ each). The extracted RNA passed the quality check for microarray analysis. The culture media were clear and immediately frozen for further analyses.

\section{NOTOXhisto Sample Processing}

For sample processing, the ground and spaceflight EUs were open and the coverslips and the spent media were collected in sterile conditions. Images of hBMSCs 2 month after fixation were obtained immediately using the inverted microscope Leica DMIL, the Leica application suite version 2.5.0R1 (20X) and the camera Leica DFC320. The spent media were kept at $-20{ }^{\circ} \mathrm{C}$.

\section{Osteogenic Differentiation Evaluation}

The osteogenic differentiation was assessed by evaluating calcium deposition using Alizarin Red staining. The Alizarin Red was extracted and measured at $405 \mathrm{~nm}$. Extraction and quantification of the dye were performed as describe previously (Gregory et al. 2004)

\section{RNA Isolation, Microarray and RNAseq Processing}

Spaceflight or $1 g$ control samples were preserved in RNAlater (Sigma-Aldrich) and suspended by scraping in $500 \mu \mathrm{L}$ of Trizol (Thermo Fisher Scientific) for total RNA extraction. 5-30 ng of high quality total RNA were used to synthesize Biotin-labelled cDNA targets. Double stranded cDNA synthesis and related cRNA was performed with
Nugen ${ }^{\circledR}$ Pico WTA Systems V2 (NuGEN Technologies, Inc). With the Encore ${ }^{\circledR}$ Biotin Module (NuGEN Technologies, Inc) was synthesized the fragmented and labelled cDNA. Hybridization was performed onto Affymetrix Gene Human 1.0 ST arrays (28.869 genes) (Affymetrix, Santa Clara, CA, USA). GeneChip arrays were scanned using an AffymetrixGeneChip ${ }^{\circledR}$ Scanner3000 7G using default parameters. AffymetrixGeneChip ${ }^{\circledR}$ Command Console software (AGCC) was used to acquire GeneChip® images and generate .DAT and .CEL files, which were used for subsequent analysis with proprietary software. Partek Genomics Suite v 6.4 was used for analysis of microarray data.

For RNAseq studies, only spaceflight samples were considered. 100ng of total RNA were used to purify mRNA and to synthesize cDNA in one tube (Thermo Fisher Scientific -Dynabeads mRNA DIRECT Micro Kit), using RCC RNA Spike-In Control Mixes (Thermo Fisher Scientific) as a control. We used the Ion Total RNA-Seq Kit v2 to construct the whole transcriptome libraries, using oligo-dT primers. Sequencing templates were prepared using an Ion PI Hi-Q OT2 200 Kit (Thermo Fisher Scientific). The prepared templates were sequenced using an Ion PI HI-Q Sequencing $200 \mathrm{Kit}$ and the Ion Proton sequencer (Thermo Fisher Scientific). Torrent Suite 4.0 to 5.0 software (Life Technologies) was used to convert the raw signals into base calls and to extract the FASTQ files of the sequencing reads.

\section{Microarray Data Analysis}

Transcriptional analyses were performed by comparing spaceflight or $1 g$ controls samples in OM vs. those in SM. We also compared spaceflight samples with $1 g$ controls in the same medium. The list of the differentially expressed genes was obtained using ANOVA. A 1.3-FC cutoff value coupled with $p<0.05$ and a false discovery rate (FDR) filter were used to distinguish statistically higher or lower gene expression from random variation. When the number of genes was $\geq 1000$, pathway analyses were performed following only a topological approach (SPIA Signaling Pathway Impact Analysis) (indicated in Fig. 4 and Supplementary Fig. 1 as:**) coming from the program Graphite (Sales et al. 2012), using indistinctly Reactome or KEGG as databases. We applied this approach since SPIA is a very severe and specific method, useful when a large amount of data is used. When the number of genes was $<1000$, the pathway analyses were performed using both topological and hypergeometric (indicated in Fig. 4 and Supplementary Fig. 1 as: *) approaches coming from the programs Graphite or FunRich (Pathan et al. 2015). The gene ontology was performed using only FunRich independently of the number of genes. 


\section{RNAseq Data Analysis}

Sickle trimmer (Joshi and Fass 2011) was used to remove low quality ends of the raw reads (using a quality threshold of 20, with option $-\mathrm{q} 20$ ) and to remove short reads (using a minimum length threshold of 30 , with option $130)$ to increase the mapping rate to reference genome. Adapters were removed using CUTADAPT (Martin 2011). Reads were mapped on the ENSEMBLE GRCh38.p5 Human Genome assembly. Genome assembly was indexed locally with long indices using command bowtie2-build in bowtie2 program (Langmead and Salzberg 2012). Reads were mapped to the reference genome following the protocol provided by Life Technologies (https://ioncommunity. thermofisher.com/docs/DOC-7062). Gene expression abundance estimation was performed with htseq count script (Anders et al. 2015). Differential expression analysis was performed using the edgeR (Robinson et al. 2010) package in R software. After library normalization, the genewise exact tests were calculated with command exactTest. Significantly differentially expressed genes between the two conditions, without adjustment for multiple testing, were identified and exported for further analysis. The pathway analyses were performed using hypergeometric test in FunRich (Pathan et al. 2015).

\section{Exosomes Purification and miRNA Analysis}

We used the exoRNeasy Serum/Plasma Midi Kit (QIAGEN S.r.l. - Milan-Italy) to extracted the exosomes and purify the miRNAs present therein, according to the manufacturer's instructions. Briefly, $800 \mu \mathrm{L}$ of spent media followed a phenol/guanidine-based lysis that allows the recovery of vesicles in a membrane-based affinity-binding step to isolate exosomes. The purification of total RNA from exosomes was possible thanks to the use of a silicamembrane. The presence and the integrity of small RNA was assessed using an Agilent Bioanalyzer (Agilent Technologies, Santa Clara, CA, USA). The samples obtained were sent to the DNA sequences services Fasteris-Switzerland (http://www.fasteris.com) for analysis by sequencing.

\section{miRNAseq Data Analysis}

Data analysis was performed by Fasteris-Switzerland. After sequencing adapters removal, performed with Trimmomatic, reads in the size range of 18-50 nucleotides were selected and used for downstream analysis. Selected sequences were mapped against mature miRNAs in the mirBase version 21 using BWA tool (command bwa aln) and miRNAs expression counts were obtained with the same tools. Finally, differential expression analysis was performed using the edgeR package in $\mathrm{R}$ software. Counts were normalized and differential expression was inferred using the negative binomial distribution and a shrinkage estimator for the distribution variance of the counts.

\section{Data Availability}

All RNA sequencing, small non-coding RNA sequencing, and Microarray data that were used to support the findings of this study have been deposited in NCBI/GEO with SuperSeries accession code GSE100933.

\section{Results}

To evaluate whether microgravity affects SC differentiation, we cultured hBMSCs in space and on Earth using suitable experiment hardware $(\mathrm{EH})$ modules with automatic fluid exchanges (Fig. 1).

Briefly, given the temperature and preservation limits of the spaceflight experiments, the cells were cultured during spaceflight for $3 \mathrm{~d}$ before docking, and then the experiment started and lasted for approximately 14 days (330 hrs) (Fig. 2) with suitable medium exchanges and final addition of suitable fixatives such as RNAlater or NOTOXhisto. There were restrictions in selecting the postflight analyses due to the use of RNAlater. At the end of the in-flight and on-Earth experiments, the cells showed the characteristic static distribution and were well attached to the culture support as determined by visual inspection using phase contrast microscopy, thus indicating that they were still alive when fixed with RNAlater (Fig. 3). The long storage time before landing had no influence on the conditions of the fixed flown samples. In conclusion, all of the SCD EHs (inflight and on Earth) were correctly activated, and cells and culture media have been fixed in accordance with the timeline. At the end, each module provided a $230-\mathrm{mm}^{2}$ cell samples and the related preserved media (media A - C, 1.8 ml each).

Photographs were taken upon opening the EUs containing cells fixed with RNAlater, using phase contrast microscopy (see example in Fig. 3-1) and showed that $\mu$ increased the production of membrane aggregates, similar to lipid vesicles but eventually attributable also to exosomal microvesicles.

The extracted RNA passed the quality check for microarray analysis ( $\mathrm{RIN}=8-10)$. The culture media were clear and immediately frozen for further analyses. All of these factors indicate that experiment ended successfully, as anticipated by the preliminary exhaustive ESTs (Experiment Sequence Test) performed on ground under ESA controls.

Here, we highlight the $\mu \mathrm{g}$ effects on hBMSCs: 1) evaluating calcium deposition; 2) adopting the genomic and 
1)

Images of hBMSCs in RNAlater

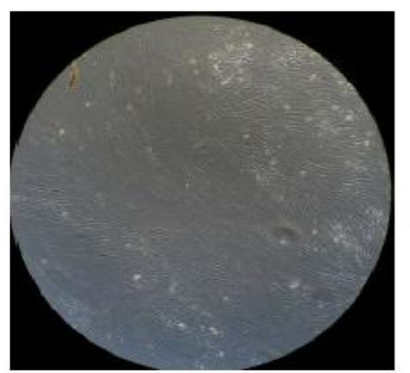

\section{Gm-OM}

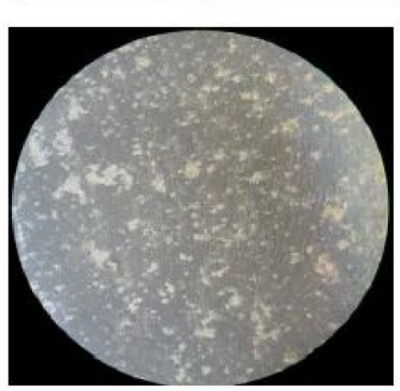

Fm-OM

\section{RNA analysis}
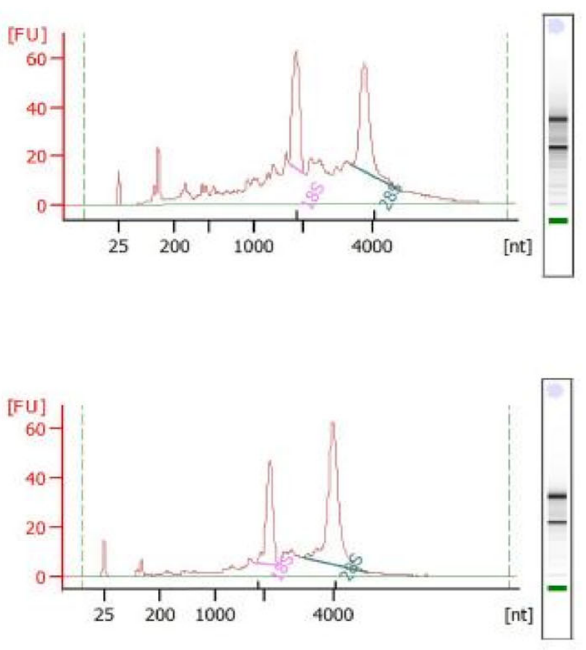

2)

A

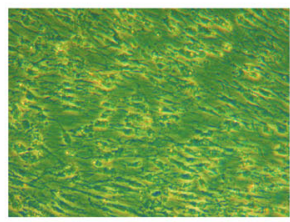

Gm-SM

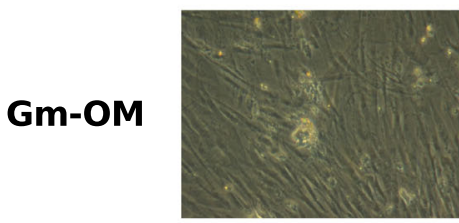

Fm-SM
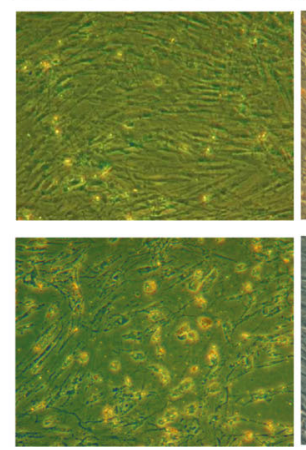

Fm-OM

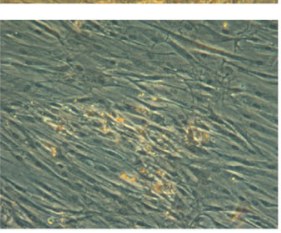

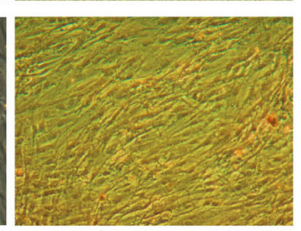

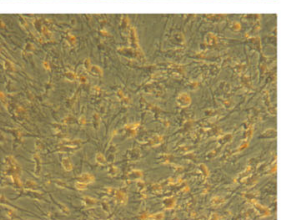

B
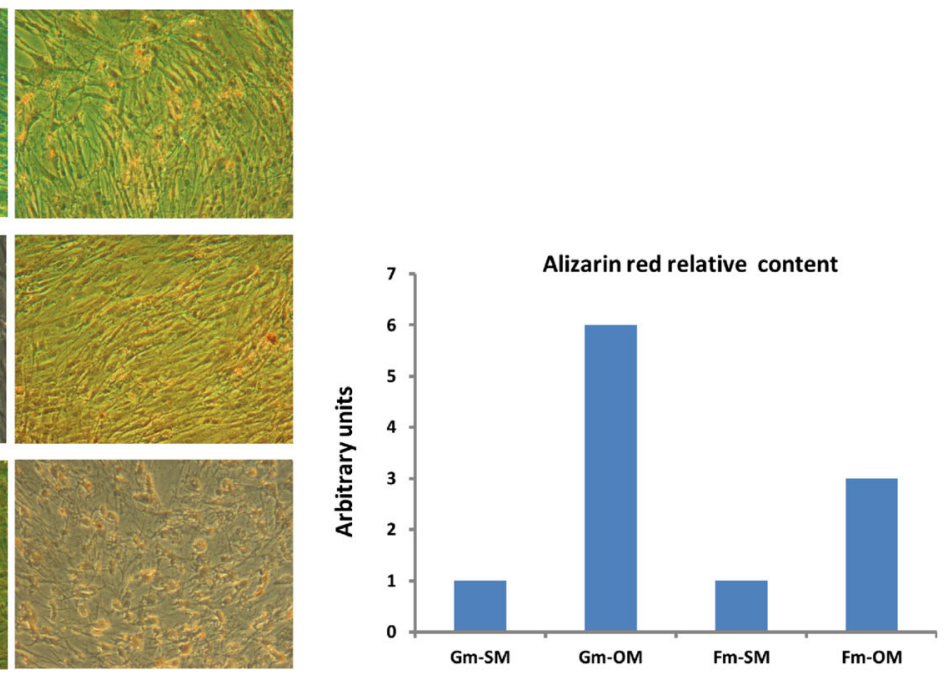

Fig. 3 SCD after the spaceflight mission. - Human BMSCs fixed in 1) RNAlater - Examples of the integrity of the samples. Selected images as obtained by Kayser - Italia after the disassembly of the EHs and related RNA analysis (Cogentech). 2) NOTOXhisto -Selected images (A) before and (B) after Alizarin red staining. Before the Launch (L), 25000 human BMSCs $/ \mathrm{mm}^{2}$ were seeded on the Thermanox supports in the 24 modules ( $12 \mathrm{Fms}$ and $12 \mathrm{Gms}$ ). Nevertheless, due to the limited number of modules, we could not determine how many cells were alive at $\mathrm{T}_{0}+7 \mathrm{hrs}$ : as a matter of fact, the 24 modules experienced the same temperature variation during the interval $\mathrm{L}-\left(\mathrm{T}_{0}+7 \mathrm{hrs}\right)$, but the 12 Fms experienced 6-8 min of hypergravity and 2-3 days of microgravity before being brought into KUBIK on ISS, while the Gms remained at $1 \mathrm{~g}$. Due to these initial differences, to evaluate calcium deposition in SM (unstimulated conditions) and in OM (Vit D3 supplemented medium, stimulated conditions) on Earth and ISS we compared the differences in alizarin red concentration [ARC] on Earth and ISS using the following ratio (arbitrary units): $[\mathrm{ARC}]_{\mathrm{T}_{0}+14 \mathrm{~d}} /[\mathrm{ARC}]_{\mathrm{T}_{0}+7 \mathrm{hrs}}$ assuming $[\mathrm{ARC}]_{\mathrm{T}_{0}+7 \mathrm{hrs}}=1$ 
the NGS approaches to validate the possible failures of the osteogenic differentiation; 3 ) focusing on the cell cycle and proliferation; 4) discussing possible evidence of stemness, senescence, apoptosis and ECM stiffness, to complete the analysis of our samples, and 5) adding other data on the presence of macrovesicles and exosomes.

\section{Calcium Deposition}

We Versari et al. (2016) have verified that the nontoxic fixative NOTOXhisto can substitute paraformaldehyde (PFA) and is suitable for preserving cell samples in spaceflight experiments. Using Alizarin Red as a marker of osteogenic differentiation, we evaluated calcium deposition by means of conventional microscopy (Fig. 3-2) In line with published data, the Alizarin Red results indicate that calcification in real $\mu g$ was much less $(<50 \%)$ than that obtained on Earth.

\section{Osteogenic Differentiation of hBMSCs in $\mu \mathrm{g}$}

The principal component analysis (PCA) of the gene expression profiles from the DNA microarrays confirmed that out of the sixteen samples fixed in RNAlater, reported in Fig. 2a, the spaceflight samples belong to clearly defined clusters (data not shown).

To investigate the genes involved in osteogenic differentiation in $\mu g$, we performed transcriptional analysis by comparing in-flight and on-Earth cells in standard (SM) or osteogenic medium $(\mathrm{OM})$.

Figure 4 and Table 1 show the relevant comparisons considered together with significant selections of the differently expressed genes (DEGs) and graphs of the most representative gene ontology (GO) and biological pathways. The comparisons are in-flight samples (Fm-OM vs. Fm-SM = comparison 1), in-flight versus ground samples (Fm-OM vs. $\mathrm{Gm}-\mathrm{OM}=$ comparison 2 and $\mathrm{Fm}-\mathrm{SM}$ vs. $\mathrm{Gm}-\mathrm{SM}=$ comparison 3). This notation will be used throughout the paper (Fig. 4-A). Moreover, a number in parenthesis to indicate its fold-change (FC) in the quoted context will often follow the gene name.

In comparison 1, we found 242 DEGs (126 downregulated, 116 upregulated). Among the upregulated, we found a few important genes associated to osteogenesis (BGLAP, $C H R D L 1$ and SPP1) but hypergeometric and topological pathway analyses indicated that most of the DEGs are associated with the GO Extracellular Matrix (ECM) and to the pathway of Extracellular Matrix Organization that was significantly inhibited (Fig. 4C). Six of the 92 genes in this pathway, that encode for type 4, 11 and 12 collagen proteins as well as for proteins that regulate collagen chain assembly, were downregulated (Bonferroni $\mathrm{p}<0.001$ ). These results agree with those of many other studies performed under conditions of $\mu g$ or sim- $\mu g$, indicating that looser interactions between collagen and integrins induce changes in ECM (Meyers et al. 2004). Thus, this confirms that osteogenic differentiation depends on the interactions between osteoblast precursors and the collagenous extracellular matrix they secrete.

We also found that four of the genes making up the pathway of Metabolism of Steroid Hormones and Vitamins $A$ and $D$ were highly up-regulated (CYP19A1, CYP24A1, $A K R 1 B 1, H S D 11 B 1)$. In particular, we found the gene CYP24Al (217.89) overexpressed, which encodes member 1 of the cytochrome P450 Family 24, an enzyme regulating the levels of vitamin D (Geng et al. 2013). This is relevant to our experiment as we used Vit D3 to induce hBMSC osteogenic differentiation. (Supplementary Tables 1-6).

To validate the data reported above, and delve more deeply in to low copy number genes, we performed the NGS analysis of the same hBMSC spaceflight samples (Fig. 5). This approach presented 480 DEGs (FC $>1.3, \mathrm{p}<0.05$ ), which is more than the 242 DEGs identified using the microarray approach. Both techniques shared 89 DEGs with concordant expression. The majority of them belong to Integrin Cell Surface Interactions pathways, confirming two important processes: osteogenesis (highly upregulated BGLAP, CHRDL1, SPP1, CYP19A1, CYP24A1, andHSD11B1) and collagen degradation (downregulated COL11A1, COL12A1, COL4A1, COLAA2, ITGA11, and THBS1), in agreement with many studies indicating $\mu g$ as major driver of changes in cytoskeleton and ECM (Louis et al. 2015; Vorselen et al. 2014) (Supplementary Tables 8-10).

The moderate osteogenic differentiation and low calcification observed in the hBMSCs in $\mu g$ is not explained by differences in expression of genes involved in apoptosis or adipogenesis: instead, they are mainly associated to the down regulation of important genes of ECM pathway.

\section{Cell Cycle and Cell Proliferation in Microgravity}

We focused on possibly important processes, other than those associated with ECM.

From microarray data, in comparison 1 we found that four genes of the LICAM Interactions pathway, involved in cell proliferation, motility and cell-cell communication (Louis et al. 2015), were down-regulated (CSNK2A2, $I T G A V, N R C A M, N R P 2)$, indicating that $\mu g$ could also affect the proliferation pattern (Supplementary Table 2). We also considered the results of the pathway analyses of comparison 2and 3 (Supplementary Fig. 1).

- In comparison 2 , we found only 6 pathways altered in $\mu \mathrm{g}$ : 5 are not directly related with cell cycle or cell proliferation (Supplementary Table 4) while the signaling by TGF- $\beta$ Receptor Complex is inhibited. The expression of theTGFB1 gene, known to cause cell cycle 
Fig. 4 Microarray results.

Microarray results analysed according to scheme $\mathbf{A}$ ) and the relative Venn diagram $\mathbf{B}$ ).

Restricted analysis focused on comparison 1 C). The GO and biological pathways were evaluated using hypergeometric approach. Results for comparison 2 and 3 are reported in Supplementary Fig. 1 a

\begin{tabular}{lcccc}
\hline & Comparison & DEGs & DEGs $\uparrow$ & DEGs $\downarrow$ \\
\hline 1 & Fm-OM vs. Fm-SM & 242 & 116 & 126 \\
2 & Fm-OM vs. Gm-OM & 5113 & 2436 & 2677 \\
3 & Fm-SM vs. Gm-SM & 5094 & 2027 & 3067 \\
\hline
\end{tabular}

b

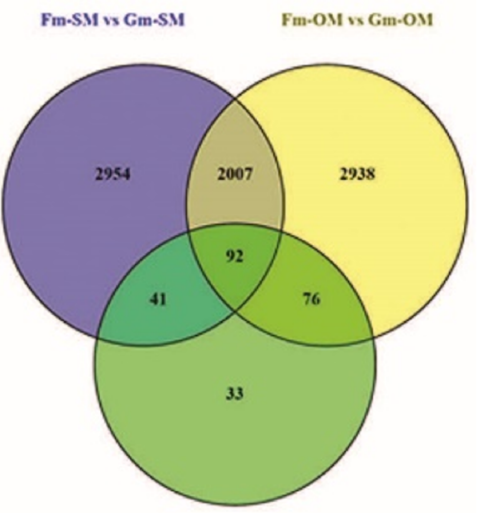

Fm-OM is Fm-SM

C

Fm-OM vs. Fm-SM
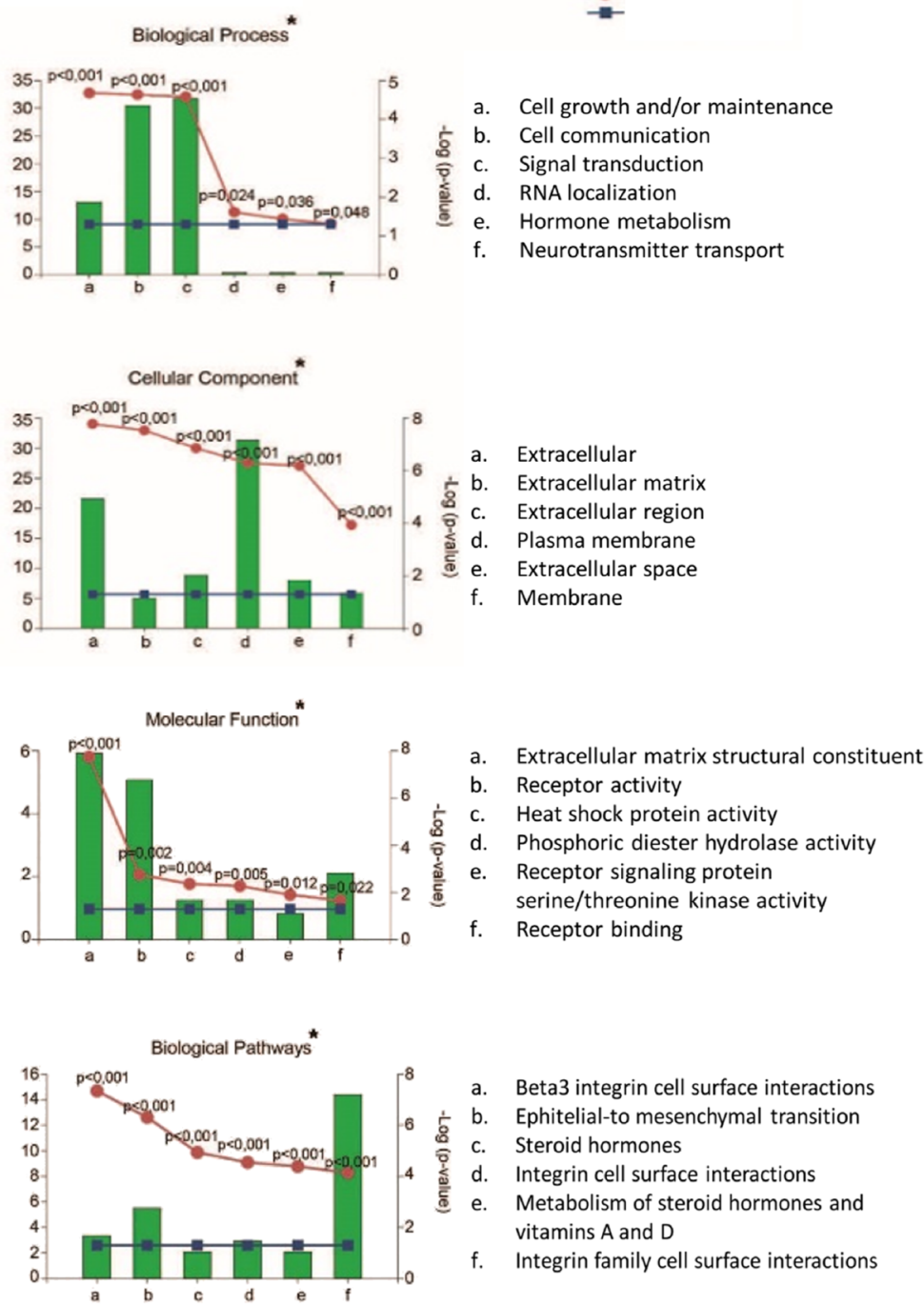
Table 1 Gene symbol and observed fold change value of relevant genes to pathway analysis reported in Fig. 5

\begin{tabular}{lr}
\hline Gene Symbol & \multicolumn{1}{l}{ FC } \\
\hline ADAMTS2 & -1.75 \\
ANKRD1 & -2.35 \\
BGLAP & -15.88 \\
COL & Mainly $\downarrow$ \\
CYP24A1 & 217.90 \\
DOCK5 & 1.38 \\
DOCK9 & -1.46 \\
FLG & -5.40 \\
G0S2 & -3.03 \\
GYS1 & -1.46 \\
IL1R1 & 2.25 \\
ITGAV & -1.67 \\
LAMA4 & 1.87 \\
LEPREL1 & -1.56 \\
NFIB & 2.33 \\
ODC1 & -1.73 \\
RAB27B & 5.23 \\
SFRP4 & -2.53 \\
\hline
\end{tabular}

arrest in $\mathrm{G1}$, was $\mathrm{FC}=1.62$; comparison 3 : $\mathrm{FC}=1.89$ (Supplementary Tables 3 and 5 respectively).

- In comparison 3, we found a) the TGFB1 gene upregulated ( $\mathrm{FC}=1.89$; Supplementary Table 5) and b) 54 altered pathways, encompassing Regulation of mitotic cell cycle, S Phase, Mitotic G1-G1/S phases, G1/S Transition, M/G1 Transition, Cyclin D associated events in G1, G1 Phase, G2/M Transition, Mitotic $G 2-G 2 / M$ phases, indicate that the cell cycle was inhibited in $\mu g$ (Supplementary Table 6). In particular, the downregulated expression of BUBI (-2.31), a serine/threonine-protein kinase that plays a central role in mitosis, and of some cyclins (CDK1 -1.80; CDKN1A -1.92; CDK6 -2.28) are in line with our hypothesis of cell cycle arrest (Dai et al. 2007; Clement 2012)
We also applied the pathway analysis to the DEGs derived from the comparison between microarray and NGS data (Fig. 5, Tables 2, 3 and 4).

- The shared DEGs between NGS and microarray were 89 and were associated to ECM integrins, Notch signalling pathway, and $\mathrm{VEGF}$ and VEGFR signalling network.

- The DEGs from NGS data, not discovered by microarrays, are 391 and were associated to the ECM integrins, and to signalling pathways, which relate to important cellular processes such as proliferation, survival and differentiation. In the case of $I L 5$ and $I L 3$ mediated signalling events (Fig. 5, Supplementary Table 10) analysis on expression of genes regulated by the signalling pathway, further stresses the implications at the cell cycle progression level. One of the genes regulated by IL5-mediated signalling events pathway is in fact PIMI $(-1.45$, NGS), which stimulates cell cycle progression at the G1-S and G2-M transitions.

- The upregulated expression of TXNIP (11.12) is considered the overall cell response to mechanic \& oxidative stresses and Vit D3 stimuli. Additionally, and perhaps more importantly, TXNIP, also known as $V D U P$, is reported to be a regulator of hematopoietic stem cell quiescence under conditions induced by Vit D3: in analogy, we assume that TXNIP over-expression was responsible of G0/G1 cell cycle arrest (Tu et al. 2016). More specifically, IL3-mediated signalling events pathway is involved in the regulation of TGFB2, and high expression levels of $T G F B 2$ in flight cells were evident from both Microarray and NGS data, suggesting that in spaceflight cell cycle arrest has occurred in the G1 phase.

- The unshared DEGs from microarray data were 153 and were associated, among others, to ECM integrins andAxon guidance (Supplementary Table 11). Those pathways are involved in reorganization of cytoskeletal and cell-cell adhesion.

Table 2 Symbol and observed fold change value of the genes shared between microarray and NGS analysis

\begin{tabular}{lcrlrrrrr}
\hline Gene symbol & Microarray FC & NGS FC & Gene symbol & Microarray FC & NGS FC & Gene symbol & Microarray FC & NGS FC \\
\hline AGT & -1.41 & -1.59 & CSF1 & 2.89 & 2.09 & VLDLR & -2.02 & -1.59 \\
BGLAP & 15.88 & 10.27 & GYS1 & -1.46 & -1.38 & NRP2 & -2.30 & -1.33 \\
CHRDL1 & 7.59 & 3.82 & JAM2 & -2.57 & -1.65 & SFRP4 & -2.53 & -1.97 \\
CLU & 3.62 & 2.68 & MAF & -1.51 & -1.33 & SOBP & 1.88 & 4.91 \\
COL11A1 & -4.28 & -3.02 & SPP1 & 9.94 & 4.02 & GHR & 2.83 & 2.56 \\
COL4A1 & -2.53 & -1.89 & SVIL & 2.09 & 1.36 & MFAP5 & -2.72 & -1.60 \\
CP & 2.24 & 1.71 & TGFB2 & 2.79 & 1.77 & NOTCH3 & -1.46 & -2.04 \\
\hline
\end{tabular}

Reported genes resulted relevant to hypergeometric pathway analysis showed in Fig. 5B 
Table 3 Symbol and observed fold change value of the genes in NGS analysis not shared by microarray analysis

\begin{tabular}{|c|c|c|c|c|c|c|c|}
\hline Gene symbol & NGS FC & Gene symbol & NGS FC & Gene symbol & NGS FC & Gene symbol & NGS FC \\
\hline $\mathrm{A} 2 \mathrm{M}$ & -1.75 & EGR3 & 6.58 & LAMC2 & 2.18 & RNF144B & -4.93 \\
\hline CCL2 & 2.28 & FGFR2 & -1.55 & LEP & -1.42 & S1PR1 & 2.57 \\
\hline CISH & -5.01 & GFRA1 & 1.98 & LIF & -1.41 & SERPINE1 & -1.84 \\
\hline COL4A3 & -2.74 & HGF & 1.96 & MAP2K6 & 8.78 & SLC2A1 & -1.58 \\
\hline COL4A4 & -2.78 & HSPB1 & -1.39 & MAPK8IP3 & -1.48 & SNTA1 & -1.48 \\
\hline COL5A1 & -1.41 & ICAM1 & -1.56 & MGP & 1.63 & THBD & 6.02 \\
\hline CSPG4 & -1.63 & IGF2 & -1.54 & NR4A1 & -2.42 & TJP2 & 1.36 \\
\hline CXCL8 & -3.27 & IGFBP1 & 1.61 & PIM1 & -1.45 & TLE4 & -1.38 \\
\hline CYCS & 1.70 & JUP & -1.41 & PITX2 & -1.39 & UBE2D1 & 1.36 \\
\hline DUSP6 & 2.18 & KIFC3 & -1.43 & PKN1 & -1.56 & DLG4 & -1.34 \\
\hline DVL1 & -1.34 & LAMA3 & 1.34 & RELB & -2.45 & HBEGF & -1.85 \\
\hline
\end{tabular}

Reported genes resulted relevant to hypergeometric pathway analysis showed in Fig. 5C

\section{Increased Microvesicle Trafficking in Microgravity}

We observed microvesicle formation in inflight samples, in particular in OM (Fig. 2-1). The microarray results of the corresponding samples (comparison 1 and 2) demonstrated that $R A B 27 b$, an important gene involved in vesiclemediated transport, was upregulated: $\mathrm{FC}=5.23$ and 7.28 respectively (Supplementary Tables 1 and 3). In addition, another 25 genes belonging to $R A B$ family were found among DEGs in comparison 2 (Supplementary Table 3).

Due to the utilization of RNAlater as fixative, it was impossible to separate exosomes and microvesicles from hBMSCs. The microvesicles/exosomes presence was indirectly confirmed when we matched the ExoCarta gene list a record of genes involved in microvesicle production and exosomal characterization (Mathivanan et al. 2012), with our microarray results. We found that 45 DEGs from comparison 2 (with 36/45 upregulated, OM) and 45 DEGs from comparison 3 (with 37/45 downregulated, SM) were matched with top 100 ExoCarta gene list. (Supplementary Table 7). Taken together, these results support our observations indicating that $\mu g$ stimulates the exosomes/microvesicle formation, being the phenomenon more evident in osteogenic conditions.

\section{MiRNAs in Exosomes from Spent Media}

To investigate if the inhibition of the cell cycle was associated to cell-cell communication through miRNAs secreted into the medium (Turturici et al. 2014), we purified exosomes from spent media and sequenced the miRNAs contained therein.

Overall, from an initial higher number of differentially expressed miRNAs, our analysis was focused on 10 miRNAs

Table 4 Symbol and observed fold change value of the genes in microarray analysis not shared by NGS analysis

\begin{tabular}{|c|c|c|c|c|c|}
\hline Gene Symbol & Microarray FC & Gene Symbol & Microarray FC & Gene Symbol & Microarray FC \\
\hline BMPR2 & -1.43 & LAMA4 & 1.87 & SRGAP1 & 1.61 \\
\hline BNIP3 & -1.44 & MAPK13 & 1.55 & $\mathrm{BNC} 2$ & 1.39 \\
\hline CCL26 & -3.82 & ODC1 & -1.73 & CALD1 & -1.34 \\
\hline CD14 & 2.93 & PLD1 & 1.56 & CRYAB & -1.44 \\
\hline CYR61 & -1.52 & THBS1 & -1.34 & FBLN1 & 1.84 \\
\hline EDIL3 & -1.81 & ZFP36 & 2.23 & MYLK & -1.81 \\
\hline EGR1 & 2.14 & ABLIM1 & -1.72 & PDGFC & -1.76 \\
\hline F2RL2 & -2.11 & ACVR2A & -2.15 & RARRES2 & 1.78 \\
\hline HIPK2 & 1.41 & APBB1IP & 1.71 & ANGPT2 & 1.61 \\
\hline IBSP & -2.73 & CSNK2A2 & -1.55 & ATP2B1 & 1.48 \\
\hline ITGA11 & -1.71 & MYH10 & -1.54 & DGKB & 1.66 \\
\hline ITGAV & -1.67 & RBPJ & -1.32 & DOCK9 & -1.46 \\
\hline KLF4 & 1.86 & RPS6KA2 & 1.48 & HABP4 & -1.37 \\
\hline
\end{tabular}

Reported genes resulted relevant to hypergeometric pathway analysis showed in Fig. 5D 
Table 5 Symbol and observed fold change value of the HSP genes in microarray analysis of comparison 2, Fm-Om vs. Gm-Om

\begin{tabular}{lc}
\hline Gene symbol & Microarray FC \\
\hline B1HASP & 2.65 \\
HSP70 family & \\
HSP1A & 2.17 \\
HSPA2 & 2.09 \\
HSPA13 & -5.41 \\
HSPA5 & -3.79 \\
HSPA9 & -3.31 \\
\hline
\end{tabular}

differentially expressed in at least one comparison, after filtration (filtering for a $\mathrm{FC} \geq 2$ and a p value, FDR corrected, $\leq 0.05$ ).

Our results (Fig. 6) are in favour of a role of miRNAs in the regulation of osteogenesis. Accordingly, other studies indicate that: a) the significant upregulation of miR-142$5 p$ correlates with active osteoblastogenesis during the bone healing process promoting in vitro osteoblast activity and matrix mineralization (Murata et al. 2014; Baglio et al. 2015); b) miR-451a is involved in the regulation of skeletal development (Scott et al. 2012; Desvignes et al. 2014) together with c) the upregulation of miR-3120-5p ( $\mathrm{Li} 2011)$.

\section{Stemness, Apoptosis and Senescence}

$\mathrm{Gm}$ and Fm samples underwent the same protocol, but the 3 days needed to reach ISS and install the EHs in the local incubator may have affected starting cell concentration, their viability and differentiation capacity (Roobol et al. 2009). Bearing in mind this caveat we evaluated the differences in genes altered between spaceflight samples

Table 6 Symbol and observed fold change value of the Linc genes in microarray analysis of comparison 2, Fm-Om vs. Gm-Om

\begin{tabular}{lc}
\hline Gene symbol & Microarray FC \\
\hline LINC00260 & 2.15 \\
LINC00266-1 & -2.10 \\
LINC00268 & 1.87 \\
LINC00471 & -2.12 \\
LINC00526 & 1.47 \\
LINC00670 & -1.62 \\
LINC00671 & 1.43 \\
LINC00943 & -3.55 \\
LINC00998 & 1.97 \\
LINC01089 & 2.68 \\
LINC01106 & 1.87 \\
LINC01116 & 1.81 \\
LINC01123 & 1.89 \\
LINC01140 & 6.19 \\
\hline
\end{tabular}

and ground controls in OM, comparison 2 (Supplementary Table 3, Supplementary Fig. 1A).

The downregulation of stemness genes, such as LIF (-44.99) and FGF2 (-5.19) points to stemness loss.

The lack of any significant sign of apoptosis (BCL2: 1.32, CASP9: -1.48 ) is supported by the upregulation of two potent inhibitors of apoptosis (BIRC5: 6.16, also known as Survivin (Saleem et al. 2013) and $I G F-1: 3.51)$ ) (Vincent and Feldman 2002).

Because of the oxidative stress associated to $\mu \mathrm{g}$, senescence could have been expected based on data arising from spaceflight experiments using other cellular models (Versari et al. 2013b; Tian et al. 2017). Considering comparison 2, we did not find any evidence of gene expression modulation of senescence-associated markers such as PARG1, CDKN2B, the growth factor PTN, MCM3, and histone deacetylases (Baker et al. 2015) except the HDAC9 $(-2,28)$. Increased expression of the transgelin gene (TAGLN: 3.55) (Wong et al. 2016) regulating the actin dynamics and stress fibers during senescence, and of TXNIP (11.21), a gene playing a positive role in preventing stem cell ageing (Jung et al. 2016), suggests a compensatory mechanism reducing senescence. This mechanism induces salvage pathways leading to production of antioxidants as indicated by the extensively increased gene expression of glutathione transferases, together with other three genes involved in the antioxidant response: GPX4 (1.70), CAT (2.70), SODl (1.72).

\section{ECM Stiffness}

A decisive parameter for osteogenesis is ECM stiffness, impossible to measure under our conditions. Nevertheless, we can deduce that many data indicate major rearrangements of ECM. They include, considering comparison 2, the high upregulation of all 12 collagen genes, the high downregulation of matrix metallopeptidases, involved in breakdown of ECM, (MMP1: -8.27; MMP3: -16.73), and the increased expression of metallopeptidase inhibitors (TIMP2: 2.57). In addition we found that lamin-A/C is overexpressed (LMNA: 5.32), in analogy with its regulators, the Retinoic acid receptors (RARs). These results are in accord with literature data (Swift et al. 2013) reporting that the majority of lamins scales with the stiffness of a tissue, with stiffer tissue such as bone having higher levels than softer tissue. The lamin genes and their regulators favour osteogenesis and impair adipogenesis.

\section{Discussion}

The delicate process of MSC differentiation is governed by physical, chemical and biological factors and numerous cell 
a NGS

Microarray

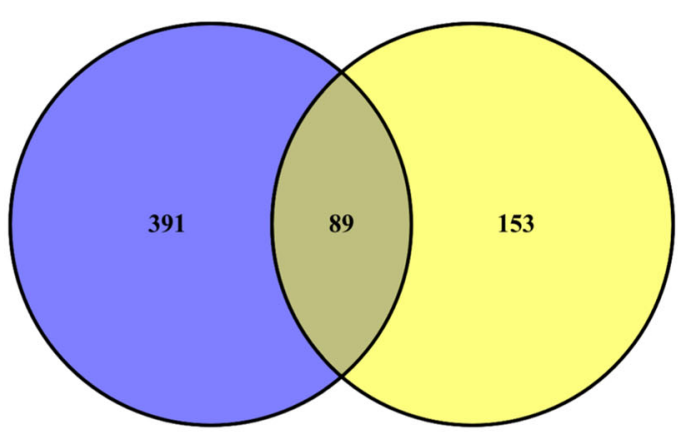

b

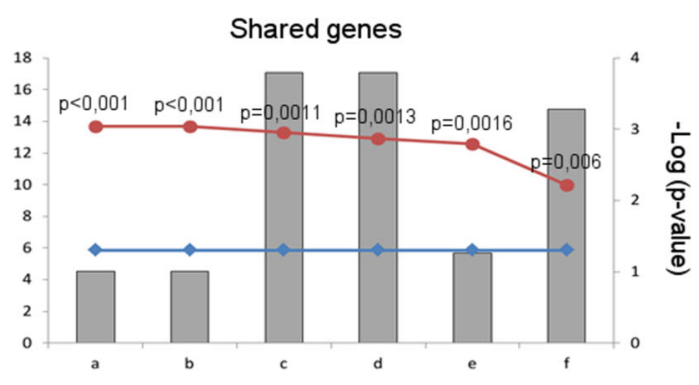

a. Notch-mediated HES/HEY network

b. Notch signaling pathway

c. Beta1 integrin cell surface interactions

d. Integrin family cell surface interactions

e. Epithelial-to-mesenchymal transition

f. VEEGF and VEGFR signaling network

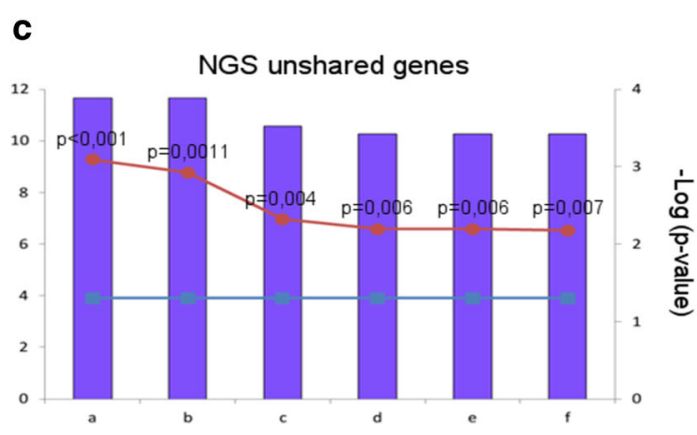

$\begin{aligned} & \% \text { of genes } \\ - & p \text {-value } \\ - & p=0.05 \text { reference }\end{aligned}$

a. Beta1 integrin cell surtace interactıons

b. Integrin family cell surface interactions

c. ErbB receptor signaling network

d. GMCSF-mediated signaling events

e. IL5-mediated signaling events

f. IL3-mediated signaling events

d

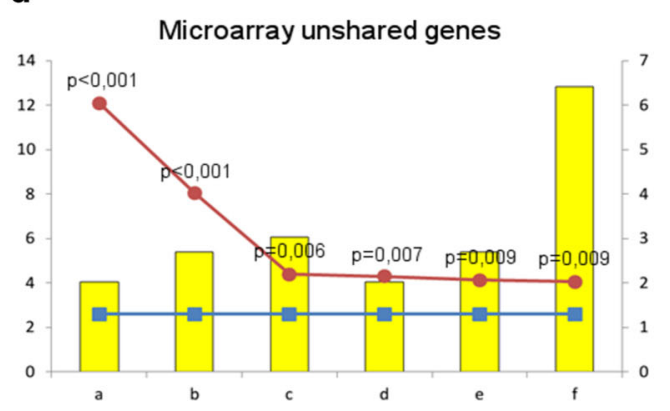

Fig. 5 Comparison of NGS and Microarray results for inflight samples A) Venn Diagram of NGS and microarray results showing the number of shared and unshared genes. Pathway analysis using a hypergeometric approach of B) NGS and microarray shared genes, C) NGS unshared genes and D) Microarray unshared genes 


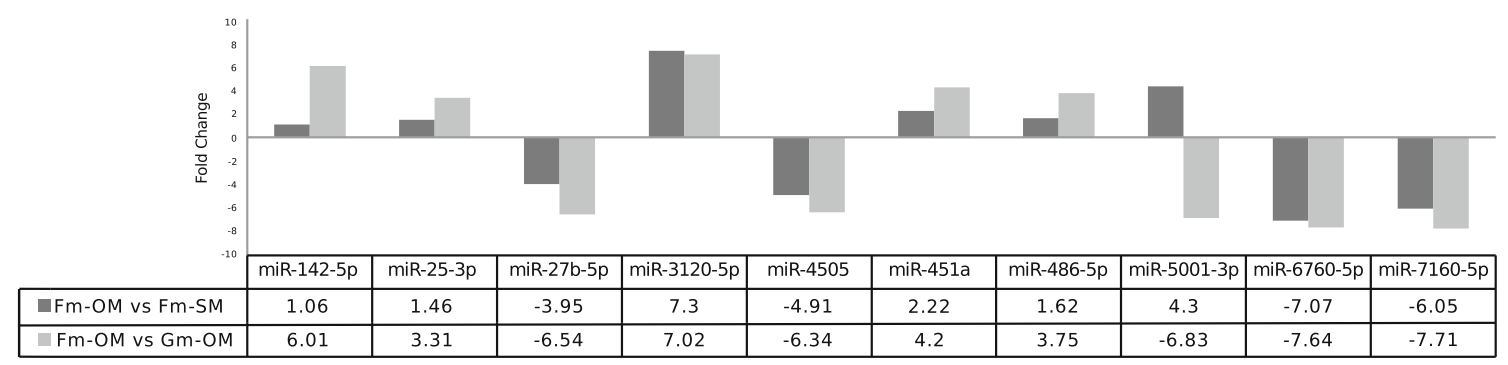

Fig. 6 List of MiRNAs released in spent media in inflight and on-ground EHs: significant comparison of their expression in SM and OM media

processes are controlled by gravity, such as calcium signalling mechanotransduction, ligand receptors interactions, and cell-cell communications.

Previous studies have indicated cytoskeletal structures, nuclei, and integrins as gravisensors, although cytoskeleton and integrins are not primary sensors since they react in response to their regulatory proteins (controllers of polymerization/destabilization agent). Consequently, it can be expected that the real $\mu g$ environment will cause massive changes with subsequent cytoskeletal and adhesion alterations.

We Versari et al. (2007, 2013a) and other authors (Chen and Jacobs 2013; Gershovich et al. 2013; Sheyn et al. 2010; Chen et al. 2016b) have used sim- $\mu g$ conditions to investigate the effects of $\mu g$ on MSCs, but considerable differences in the experimental conditions, simulating devices, bioreactors, media, precursor cellular models etc. mean that many sim- $\mu g$ results cannot be compared with those derived from the SCD experiment. In addition, we chose Vit D3 as our osteogenic stimulus, with the aim of producing in vitro and in $\mu \mathrm{g}$ results comparable to those obtained in mammalian models in space (Smith et al. 2012), whereas the most widely used chemical in in vitro MSCs osteogenic differentiation is Dexamethasone (DEX) (Bradamante et al. 2014, and references therein). In detail, Vit D3 (but not DEX): (a) stabilizes its own receptor, Vitamin D receptor (VDR) and induces the expression of

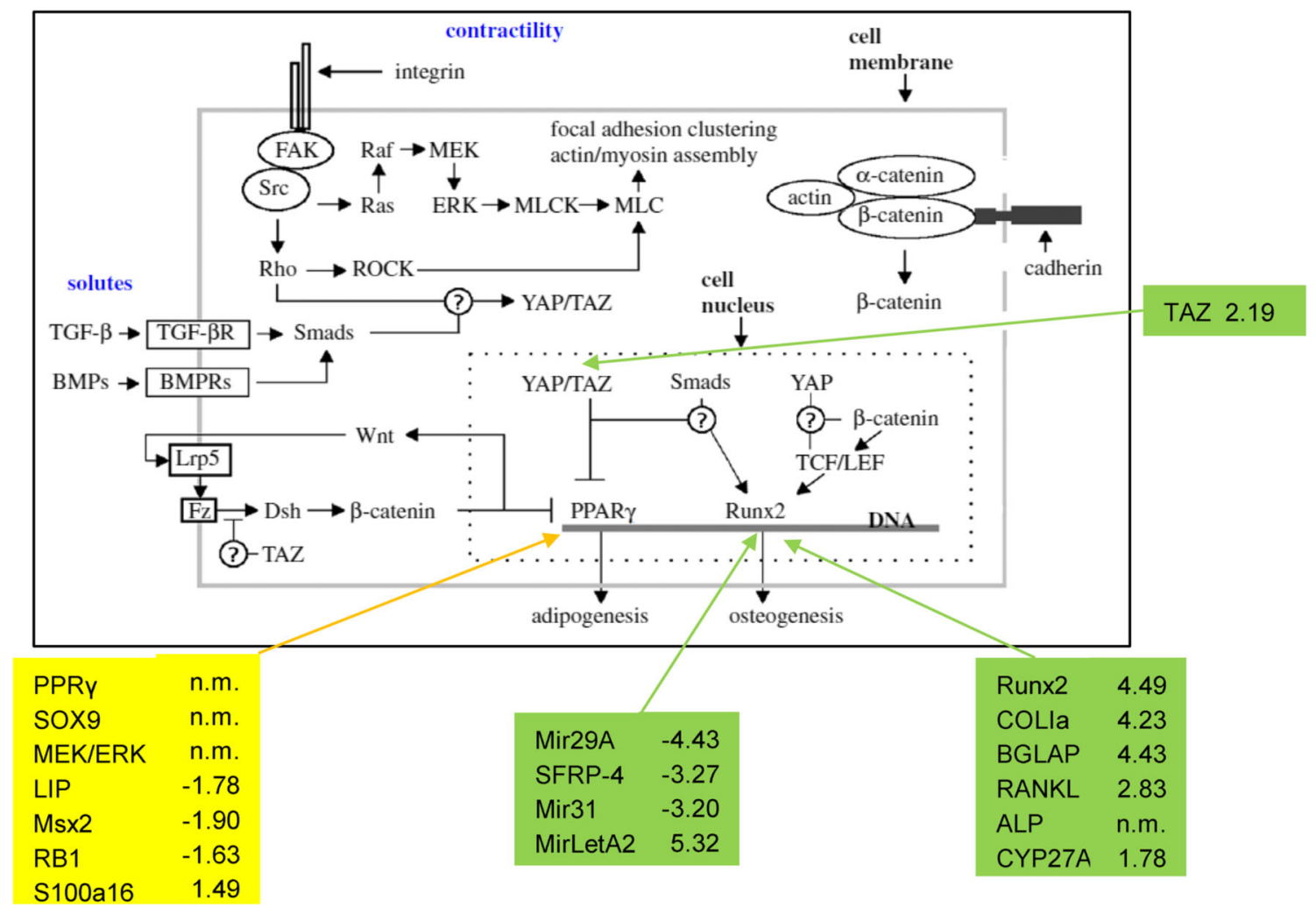

Fig. 7 A simplified scheme of signaling pathways implicated in MSC mechanosensing, mechanotransduction, and differentiation (adapted from MacQueen et al., 2013). From comparison 2, Fm-OM vs. Gm$\mathrm{OM}$, microarray analysis, The figure highlights some important DEGs associated in coloured frameworks because of their known functions: green: Taz and osteogenesis modulators; yellow: involved in lipid production. Numbers indicate their expression 
bone morphogenetic protein-2 (BMP-2) during osteoblast differentiation; (b) inhibits adipocyte differentiation in murine bMSCs and inhibits in the TMS-14 line of preadipocytes that support osteoclast-like cell formation.

Gene expression data points to osteoblast formation, rather than the formation of adipocytes (no modulated expression of $P P R \gamma, S O X 9$ and $M E K / E R K$ ) or chondrocytes (no modulated expression of SOX9 and PI3K/ATK).

Mechanical signals (substrate stiffness, cell shape, fluid flow, compression, tension etc.) are key regulators of MSC differentiation: these signals are detected by mechanosensors, and transformed into biological reactions whose result are transmitted to the cell nucleus, thus leading to alterations in gene expression and protein activity, that may also be responsible for extracellular changes. Cell membranes allocate many components acting as mechanosensors, in particular ion channels, integrin, cadherin, and primary cilia, the last ones acting as chemo and mechanosensors.

Integrins are considered the main mechanosensors in many cells, including MSCs (Liu et al. 2014). They are also a link between extracellular and intracellular environment as they bind with ECM extracellularly, and promote the formation of focal adhesion complexes intracellularly. Focal adhesion proteins act as actin-integrin anchorage points for the cytoskeleton, and are involved in many signalling pathways.

Going from $1 g$ to $\mu g$ (see comparison 2 and 3) many genes of integrins became upregulated, especially under osteogenic conditions. The encoded focal adhesion proteins function as messengers in the signal transduction pathway mediating adhesion and modulating the cytoskeletal organization of actin bundles. The Zyxin gene encoding a protein described as tension sensor (Luis Alonso and Goldmann 2016) is in our case slightly modulated (1.47), but the expected changes in cell volume and channel activation are confirmed by the upregulation of the $\mathrm{K}^{+}$stretchactivated ion channel (KCND2: 4.33 and KCNK2: 4.83). Today, in both normal physiology and diseased conditions the mechanosensitive cation channels Piezo1 and Piezo2 linked to integrins, "are considered at the centre-stage of the future mechanobiology". Interestingly, we found an increased gene expression of PIEZO2 (4.07, comparison 2) at the end of the SCD experiment (Nourse and Pathak 2017).

Although major actin modifications were expected in the spaceflight samples, we did not observe them, because, in our opinion, by the end of the SCD experiment $(\sim 400$ hours after the launch) the hBMSC cell culture had already adapted to space conditions. Indeed, the cytoskeletal modifications induced by the short period of hyper- $g$ after the launch as well as by the subsequent few hours needed to reach the orbit are evident after a short time interval (Vorselen et al. 2014). Nevertheless, we found the expression of many genes that encode for HSPs modulated (Table 5). Differently, osteogenesis is a slow process that lasts not for seconds but days on ground (Kulterer et al. 2007), the two weeks of the SCD experiment were dictated by the preceding experimental evidences (Versari et al. 2016).

Signalling pathways involved in contractility-based mechanosensing and MSC proliferation/differentiation (Bradamante et al. 2014) underline the central role of the YAP/TAZ system, (Yes-Associated Protein and Transcriptional coActivator with PGZ-binding motif) effectors of the Hippo pathway in modulating cell proliferation or differentiation (Abdallah et al. 2015; Halder et al. 2012).

Since factors involved in determining BMSC commitment to osteoblasts or adipocytes have been reviewed by others (Abdallah et al. 2015; Kokabu et al. 2016) and since this is not the aim of this study, even if we have obtained results consistent with the literature conclusions, we will not discuss the data obtained comparing Gm-OM vs Gm-SM experiments.

It is known that alteration in stiffness, in terms of membrane stretching and its adhesion complex and cytoskeletal changes, implicates also nucleus and chromatin alterations (Graham and Burridge 2016). In addition, only a rigid ECM keeps YAP/TAZ active at the nucleus (Piccolo et al. 2014; Matsumoto et al. 2016; Cosgrove et al. 2016). In our case where membrane stiffness and cytoskeleton have been severely modified by $\mu \mathrm{g}$, the YAP/TAZ system responded to the abovementioned changes with limited increased TAZ gene expression (WWTRl: 2.19) and altered gene expression of some inhibitors and activators. In addition, the Linc complex (Table 6), essential for cytoskeletal strain transfer to the nucleus and activation of YAP/TAZ process (Driscoll et al. 2015), shows extensive altered gene expression.

Here we summarize some features, reported in Fig. 7 (MacQueen et al. 2013), that are in favour of osteoblastogenesis.

We observed the upregulated expression of main osteogenic genes (RUNX2: 4.49; COLIa: 4.23), osteocalcin, and a marker of late differentiation (BGLAP: 4.42). The $A L P$ gene, an index of early osteo differentiation was not differentially expressed, thus suggesting that the analysed samples were already in stand-by. The activation of RUNX2 agrees with the downregulation of MIR31 (-3.20), which is known to lead to inhibition of osteoblast formation (Baglìo et al. 2013).

Even if some genes expected to increase osteoblastogenesis and decrease adipogenesis (Kokabu et al. 2016) were found modulated favouring adipogenesis, we found important genes like MIRLET7A2 (5.32), known to enhance osteogenesis while repressing adipogenesis (Wei et al. 2014), highly upregulated. In favour of osteogenesis, there is also the evidence of modulation of the Canonical (WNT3b 
and WNT10b: n.m.; Lrp5: 2.28, Fz mainly upregulated, DDK1 (inhibitor): 4.89) and non-Canonical Wnt signalling (WNT5a: -3.21, WNT5b: n.m).

Nevertheless, it is relevant that the upregulation of the inhibitor of early differentiationHOXA2 (4.77) and the down regulation of IL1 (Sonomoto et al. 2012) (ILla: -49.30; ILIb: -25.80) should impair differentiation.

\section{Conclusion}

The SCD - Stem Cell Differentiation experiment demonstrates that hBMSCs are affected by $\mu g$ and respond to $\mu g$ stresses reverting to a quiescent state (Winer et al. 2009) after a moderate osteogenic differentiation. We indicate that this condition could be reversible due to the upregulation of some decisive miRNAs present in exosomes. Our results are in line with experiments indicating that BMSC differentiation in $\mu g$-exposed mice resulted in increased osteoblastogenesis potential following reloading at $1 \mathrm{~g}$ (Blaber et al. 2014; Blaber et al. 2015). Despite a number of limitations due to the well-known inconveniences of space biology, from the limited number of samples to the numerous operational constrains, our results also indicate that many different responses are activated to compensate for the initial $\mu \mathrm{g}$ shock, to adapt hBMSCs to the needs of survival and to differentiation stimuli. Based on gene expression data, cell cycle arrest occurs after an initial differentiation process with no evidence for normal terminal differentiation markers as they are expressed in mechanically loaded tissues.

Finally, it is remarkable that some of the genes identified as "specific space genes" (Abdelmoaty et al. 2015) were highly modulated in our SCD experiment. Among the specific genes indicated as associated to $\mu \mathrm{g}$ in different cellular models, we found TXNIP,EGR1, FN, integrins, $K P N A 2, M T$, and $T U B$ as common in the DEG lists of NSG and microarray analyses.

After more than two weeks in $\mu g$, we did not find indication of adipogenesis or apoptosis, and senescence from our genome-wide and NGS analyses. We hypothesize that the activation of salvage and compensatory pathways leading to glutathione production could provide a hermetic effect, such as that induced by mild stressors, resveratrol, that leads to improved protection from stress. This is in agreement with the fact that, unpredictably, our results on PinX1 and Survivin gene expression are in line with the first findings of NASA Twins Study (https://www.nasa.gov/twins-study) concerning transient differences in the twins' DNA and in particular, larger chromosomes after space travel, eventually supporting the hope that space flight could reduce aging.

Acknowledgments The authors thank the European Space Research and Technology Centre (ESTEC) Life Science Instrumentation Laboratory, (USOC BIOTESC, Hergiswil-Luzern, Switzerland); Kayser
Italia (Livorno, Italy); S. P. Korolyov Rocket and Space Corp. Energia (Moscow, Russia); and European Space Agency (ESA) Office (Moscow, Russia) for technical and logistic support. The authors thank also Jack J.W.A van Loon and Silvia Versari for their scientific assistance. The project was financially supported by the ESA-ESTEC (contracts N. 4200014651/SH/NH/NL CCN 003 and CCN 004) and by the Italian Space Agency (ASI).

Author Contributions S.B. conceived the project, designed the experiments and directed the work. D.R and M.B. performed experiments. S.M. and F.V. conducted all the bioinformatics analyses. D.R., L.B. and D.C. discussed the data. S.B., L.B. and D.C. wrote the manuscript.

\section{Compliance with Ethical Standards}

Conflict of interests The authors declare no conflict of interest.

Abbreviations AT-MSCs, Adipose Tissue derived Mesenchymal Stem Cells; BMSCs, Bone Marrow Mesenchymal Stem Cells; CC, Culture Chamber; EU, Experimental Unit; ESA, European Space Agency; EH, Experiment Hardware; FM, Flight Model; GM, Ground Model; hBMSCs, human bone marrow mesenchymal stem cells; ISS, International Space Station; MSCs, Mesenchymal Stem Cells; $\mu \mathrm{g}$, Microgravity; NGS, Next Generation Sequencing; OM, Osteogenic Medium; sim- $\mu$ g, Simulated microgravity; SCs, Stem Cells; SM, Standard Medium; Vit, D3 1,25-dihydroxy vitamin D3.

Open Access This article is distributed under the terms of the Creative Commons Attribution 4.0 International License (http:// creativecommons.org/licenses/by/4.0/), which permits unrestricted use, distribution, and reproduction in any medium, provided you give appropriate credit to the original author(s) and the source, provide a link to the Creative Commons license, and indicate if changes were made.

\section{References}

Abdallah, B.M., Jafari, A., Zaher, W., et al.: Skeletal (stromal) stem cells: an update on intracellular signaling pathways controlling osteoblast differentiation. Bone 70, 28-36 (2015). https://doi.org/10.1016/j.bone.2014.07.028

Abdelmoaty, H., Hammond, T.G., Wilson, B.L., et al.: Identification of Putative Major Space Genes Using Genome-Wide Literature Data. https://doi.org/10.5772/60412 (2015)

Adams, G.R., Caiozzo, V.J., Baldwin, K.M.: Skeletal muscle unweighting: spaceflight and ground-based models. J. Appl. Physiol. 95, 2185-201 (2003). https://doi.org/10.1152/japplphysiol. 00346.2003

Anders, S., Pyl, P.T., Huber, W.: HTSEq-a Python framework to work with high-throughput sequencing data. Bioinformatics 31, 166-169 (2015). https://doi.org/10.1093/bioinformatics/btu638

Arfat, Y., Xiao, W.-Z., Iftikhar, S., et al.: Physiological effects of microgravity on bone cells. Calcif. Tissue Int. 94, 569-579 (2014). https://doi.org/10.1007/s00223-014-9851-x

Baglìo, S.R., Devescovi, V., Granchi, D., Baldini, N.: MicroRNA expression profiling of human bone marrow mesenchymal stem cells during osteogenic differentiation reveals Osterix regulation by miR-31. Gene 527, 321-331 (2013). https://doi.org/10.1016/j. gene.2013.06.021

Baglio, S.R., Rooijers, K., Koppers-Lalic, D., et al.: Human bone marrow- and adipose-mesenchymal stem cells secrete exosomes enriched in distinctive miRNA and tRNA species. Stem. Cell Res. Ther. 6, 127 (2015). https://doi.org/10.1186/s13287-015-0116-Z 
Baker, N., Boyette, L.B., Rocky, S., et al.: Characterization of bone marrow-derived mesenchymal stem cells in aging. Bone 70, 37-47 (2015). https://doi.org/10.1016/j.bone.2014.10.014

Blaber, E.A., Dvorochkin, N., Torres, M.L., et al.: Mechanical unloading of bone in microgravity reduces mesenchymal and hematopoietic stem cell-mediated tissue regeneration. Stem. Cell Res. 13, 181-201 (2014). https://doi.org/10.1016/j.scr.2014.05.005

Blaber, E.A., Finkelstein, H., Dvorochkin, N., et al.: Microgravity reduces the differentiation and regenerative potential of embryonic stem cells. Stem. Cells Dev. 24, 2605-21 (2015). https://doi.org/10.1089/scd.2015.0218

Bradamante, S., Barenghi, L., Maier, J.: Stem cells toward the future: the space challenge. Life 4, 267-280 (2014). https://doi.org/10. 3390/life4020267

Cazzaniga, A., Maier, J.A.M., Castiglioni, S.: Impact of simulated microgravity on human bone stem cells: new hints for space medicine. Biochem. Biophys. Res. Commun. 473, 181-186 (2016). https://doi.org/10.1016/j.bbrc.2016.03.075

Chen, J.C., Jacobs, C.R.: Mechanically induced osteogenic lineage commitment of stem cells. Stem. Cell Res. Ther. 4, 107 (2013). https://doi.org/10.1186/scrt318

Chen, Q., Shou, P., Zheng, C., et al.: Fate decision of mesenchymal stem cells: adipocytes or osteoblasts? Cell Death Differ. 23, 1128-39 (2016a). https://doi.org/10.1038/cdd.2015.168

Chen, Z., Luo, Q., Lin, C., et al.: Simulated microgravity inhibits osteogenic differentiation of mesenchymal stem cells via depolymerizing F-actin to impede TAZ nuclear translocation. Sci. Rep. 6, 30322 (2016b). https://doi.org/10.1038/srep30322

Clement, J.Q.: Gene expression microarrays in microgravity research: toward the identification of major space genes, INTECH Open Access Publisher. https://doi.org/10.5772/28693 (2012)

Cosgrove, B.D., Mui, K.L., Driscoll, T.P., et al.: N-cadherin adhesive interactions modulate matrix mechanosensing and fate commitment of mesenchymal stem cells. Nat. Mater. 15, 12971306 (2016). https://doi.org/10.1038/nmat4725

Dai, Z.-Q., Wang, R., Ling, S., Wan, Y., Li, Y.: Simulated microgravity inhibits the proliferation and osteogenesis of rat bone marrow mesenchymal stem cells. Cell Prolif. 40(5), 671-684 (2007). https://doi.org/10.1111/j.1365-2184.2007.00461.x

Desvignes, T., Contreras, A., Postlethwait, J.H.: Evolution of the miR199-214 cluster and vertebrate skeletal development. RNA Biol. 11, 281-294 (2014). https://doi.org/10.4161/rna.28141

Driscoll, T.P., Cosgrove, B.D., Heo, S.-J., et al.: Cytoskeletal to nuclear strain transfer regulates YAP signaling in mesenchymal stem cells. Biophys. J. 108, 2783-93 (2015). https://doi.org/10.1016/j.bpj. 2015.05.010

Geng, S., Zhou, S., Bi, Z., Glowacki, J.: Vitamin D metabolism in human bone marrow stromal (mesenchymal stem) cells. Metabolism 62, 768-77 (2013). https://doi.org/10.1016/j.metabol. 2013.01.003

Gershovich, P.M., Gershovich, Y.G., Buravkova, L.B.: Molecular genetic features of human mesenchymal stem cells after their osteogenic differentiation under the conditions of microgravity. Human Physiol. 39, 540-544 (2013). https://doi.org/10.1134/S0 36211971305006X

Eyckmans, J., Lin, G.L., Chen, C.S.: Adhesive and mechanical regulation of mesenchymal stem cell differentiation in human bone marrow and periosteum-derived progenitor cells. Biol Open 1, 10581068. Hum. Physiol. 39, 540-544 (2012). https://doi.org/10.1134/ S036211971305006X

Graham, D.M., Burridge, K.: Mechanotransduction and nuclear function. Curr. Opin. Cell Biol. 40, 98-105 (2016). https://doi.org/10. 1016/j.ceb.2016.03.006

Gregory, C.A., Grady Gunn, W., Peister, A., Prockop, D.J.: An Alizarin red-based assay of mineralization by adherent cells in culture: comparison with cetylpyridinium chloride extraction. Anal.
Biochem. 329, 77-84 (2004). https://doi.org/10.1016/j.ab.2004. 02.002

Grimm, D., Grosse, J., Wehland, M., et al.: The impact of microgravity on bone in humans. Bone 87, 44-56 (2016). https://doi.org/10.1016/j.bone.2015.12.057

Halder, G., Dupont, S., Piccolo, S.: Transduction of mechanical and cytoskeletal cues by YAP and TAZ. Nat. Rev. Mol. Cell Biol. 13, 591-600 (2012). https://doi.org/10.1038/nrm3416

Huang, C., Dai, J., Zhang, X.A.: Environmental physical cues determine the lineage specification of mesenchymal stem cells. Biochim. Biophys. Acta 1850, 1261-6 (2015). https://doi.org/10. 1016/j.bbagen.2015.02.011

Ingber, D.E., Wang, N., Stamenović, D.: Tensegrity, cellular biophysics, and the mechanics of living systems. Rep. Prog. Phys. 77, 46603 (2014). https://doi.org/10.1088/0034-4885/77/4/046603

Joshi, N., Fass, J.: Sickle: a sliding-window, adaptive, quality-based trimming tool for FastQ files (version 1.33). [Software]. https:// www.githubcom/najoshi/sickle (2011)

Jung, H., Kim, D.O., Byun, J.-E., et al.: Thioredoxin-interacting protein regulates haematopoietic stem cell ageing and rejuvenation by inhibiting p38 kinase activity. Nat. Commun. 7, 13674 (2016). https://doi.org/10.1038/ncomms13674

Kokabu, S., Lowery, J.W., Jimi, E.: Cell fate and differentiation of bone marrow mesenchymal stem cells. Stem. Cells Int. 2016, 1-7 (2016). https://doi.org/10.1155/2016/3753581

Kulterer, B., Friedl, G., Jandrositz, A., et al.: Gene expression profiling of human mesenchymal stem cells derived from bone marrow during expansion and osteoblast differentiation. BMC Genom. 8, 70 (2007). https://doi.org/10.1186/1471-2164-8-70

Langmead, B., Salzberg, S.L.: Fast gapped-read alignment with Bowtie 2. Nat. Methods 9, 357-359 (2012). https://doi.org/10.1038/nmeth.1923

Li, J.: Quiescence regulators for hematopoietic stem cell. Exp. Hematol. 39, 511-520 (2011). https://doi.org/10.1016/j.exphem.2011. 01.008

Lian, J.B., Stein, G.S., van Wijnen, A.J., et al.: MicroRNA control of bone formation and homeostasis. Nat. Rev. Endocrinol. 8, 212-27 (2012). https://doi.org/10.1038/nrendo.2011.234

Liu, L., Zong, C., Li, B., et al.: The interaction between $\beta 1$ integrins and ERK1/2 in osteogenic differentiation of human mesenchymal stem cells under fluid shear stress modelled by a perfusion system. J. Tissue Eng. Regen. Med. 8, 85-96 (2014). https://doi.org/10.1002/term.1498

Louis, F., Deroanne, C., Nusgens, B., et al.: RhoGTPases as key players in Mammalian cell adaptation to microgravity. Biomed. Res. Int. 2015, 1-17 (2015). https://doi.org/10.1155/2015/747693

Luis Alonso, J., Goldmann, H.W.: Cellular mechanotransduction. AIMS Biophys. 3, 50-62 (2016). https://doi.org/10.3934/biophy. 2016.1.50

Luna, C., Yew, A.G., Hsieh, A.H.: Effects of angular frequency during clinorotation on mesenchymal stem cell morphology and migration. npj Microgravity 1, 15007 (2015)

MacQueen, L., Sun, Y., Simmons, C.A.: Mesenchymal stem cell mechanobiology and emerging experimental platforms. J. R. Soc. Interface 10, 20130179 (2013). https://doi.org/10.1098/rsif.2013. 0179

Martin, M.: Cutadapt removes adapter sequences from highthroughput sequencing reads. EMBnet. J. 17, 10 (2011). https:// doi.org/10.14806/ej.17.1.200

Mathivanan, S., Fahner, C.J., Reid, G.E., Simpson, R.J.: Exocarta 2012: database of exosomal proteins, RNA and lipids. Nucleic Acids Res. 40, D1241-D1244 (2012). https://doi.org/10.1093/ nar/gkr828

Matsumoto, Y., La Rose, J., Kent, O.A., et al.: Reciprocal stabilization of $\mathrm{ABL}$ and $\mathrm{TAZ}$ regulates osteoblastogenesis through transcription factor RUNX2. J. Clin. Invest. 126, 4482-4496 (2016). https://doi.org/10.1172/JCI87802 
McBeath, R., Pirone, D.M., Nelson, C.M., Bhadriraju, K., Chen, C.S.: Cell shape, cytoskeletal tension, and RhoA regulate stem cell lineage commitment. Dev. Cell 6, 483-495 (2004)

Meyers, V.E., Zayzafoon, M., Gonda, S.R., et al.: Modeled microgravity disrupts collagen I/integrin signaling during osteoblastic differentiation of human mesenchymal stem cells. J. Cell Biochem. 93, 697-707 (2004). https://doi.org/10.1002/jcb.20229

Murata, K., Yoshitomi, H., Furu, M., et al.: MicroRNA-451 down-regulates neutrophil chemotaxis via p38 MAPK. Arthritis Rheumatol. 66, 549-559 (2014). https://doi.org/10.1002/art.38269

Nagaraja, M.P., Risin, D.: The current state of bone loss research: data from spaceflight and microgravity simulators. J. Cell Biochem. 114, 1001-1008 (2013). https://doi.org/10.1002/jcb.24454

Nourse, J.L., Pathak, M.M.: How cells channel their stress: interplay between Piezo1 and the cytoskeleton. Semin. Cell. Dev. Biol. 71, 3-12 (2017). https://doi.org/10.1016/j.semcdb.2017.06.018

Pathan, M., Keerthikumar, S., Ang, C.-S., et al.: Funrich: An open access standalone functional enrichment and interaction network analysis tool. Proteomics 15, 2597-2601 (2015). https://doi.org/10.1002/pmic.201400515

Piccolo, S., Dupont, S., Cordenonsi, M.: The biology of YAP/TAZ: hippo signaling and beyond. Physiol. Rev. 94, 1287-312 (2014). https://doi.org/10.1152/physrev.00005.2014

Robinson, M.D., McCarthy, D.J., Smyth, G.K.: edgeR: a Bioconductor package for differential express ion analysis of digital gene expression data. Bioinformatics 26, 139-140 (2010). https://doi.org/10.1093/bioinformatics/btp616

Roobol, A., Carden, M.J., Newsam, R.J., Smales, C.M.: Biochemical insights into the mechanisms central to the response of mammalian cells to cold stress and subsequent rewarming. FEBS J. 276, 286302 (2009). https://doi.org/10.1111/j.1742-4658.2008.06781.x

Saleem, M., Qadir, M.I., Perveen, N., et al.: Inhibitors of apoptotic proteins: New targets for anticancer therapy. Chem. Biol. Drug Des. 82, 243-251 (2013). https://doi.org/10.1111/cbdd.12176

Sales, G., Calura, E., Cavalieri, D., Romualdi, C.: Graphite - a Bioconductor package to convert pathway topology to gene network. BMC Bioinforma. 13, 20 (2012). https://doi.org/10.1186/14712105-13-20

Scott, H., Howarth, J., Lee, Y.B., et al.: Mir-3120 is a mirror microRNA that targets heat shock cognate protein 70 and auxilin messenger RNAs and regulates clathrin vesicle uncoating. J. Biol. Chem. 287, 14726-33 (2012). https://doi.org/10.1074/jbc.M111. 326041

Sheyn, D., Pelled, G., Netanely, D., et al.: The effect of simulated microgravity on human mesenchymal stem cells cultured in an osteogenic differentiation system: a bioinformatics study. Tissue Eng. Part A 16, 3403-12 (2010). https://doi.org/10.1089/ ten.tea.2009.0834

Smith, S.M., Heer, M.A., Shackelford, L.C., et al.: Benefits for bone from resistance exercise and nutrition in long-duration spaceflight: evidence from biochemistry and densitometry. J. Bone Miner. Res. 27, 1896-1906 (2012). https://doi.org/10.1002/jbmr.1647

Sonomoto, K., Yamaoka, K., Oshita, K., et al.: Interleukin-1 $\beta$ induces differentiation of human mesenchymal stem cells into osteoblasts via the Wnt-5a/receptor tyrosine kinase-like orphan receptor 2 pathway. Arthritis Rheum. 64, 3355-3363 (2012). https://doi.org/10.1002/art.34555

Swift, J., Ivanovska, I.L., Buxboim, A., et al.: Nuclear lamin-A scales with tissue stiffness and enhances matrix-directed differentiation. Science 341, 1240104 (2013). https://doi.org/10.1126/science.124 0104

Tian, Y. et al.: The impact of oxidative stress on the bone system in response to the space special environment. Int. J. Mol. Sci. 18, 2132 (2017)

Turturici, G., Tinnirello, R., Sconzo, G., Geraci, F.: Extracellular membrane vesicles as a mechanism of cell-to-cell communication: advantages and disadvantages. Amer. J. Physiol.-Cell Physiol. 306, C621-C633 (2014)

van Deursen, J.M.: The role of senescent cells in ageing. Nature 509, 439-46 (2014). https://doi.org/10.1038/nature13193

Vernikos, J., Schneider, V.S.: Space, gravity and the physiology of aging: parallel or convergent disciplines? A mini-review. Gerontology 56, 157-66 (2010). https://doi.org/10.1159/000252852

Versari, S., Klein-Nulend, J., van Loon, J., Bradamante, S.: Influence of oxygen in the cultivation of human mesenchymal stem cells in simulated microgravity: an explorative study. Microgravity Sci. Technol. 25, 59-66 (2013a). https://doi.org/10.1007/s12217-0129333-6

Versari, S., Longinotti, G., Barenghi, L., et al.: The challenging environment on board the International Space Station affects endothelial cell function by triggering oxidative stress through thioredoxin interacting protein overexpression: the ESA-SPHINX experiment. FASEB J. 27, 4466-4475 (2013b). https://doi.org/10.1096/fj.13-229195

Versari, S., Barenghi, L., van Loon, J., Bradamante, S.: The SCD stem cell differentiation ESA project: Preparatory work for the spaceflight mission. Microgravity Sci. Technol. 28, 19-28 (2016). https://doi.org/10.1007/s12217-015-9466-5

Versari, S., Villa, A., Helder, M.N., et al.: Effects of gravity on proliferation and differentiation of adipose tissue-derived stem cells. J. Gravit. Physiol. 14, P127-P128 (2007)

Vico, L., Collet, P., Guignandon, A., et al.: Effects of long-term microgravity exposure on cancellous and cortical weight-bearing bones of cosmonauts. Lancet (Lond. England) 355, 1607-1611 (2000)

Vico, L., van Rietbergen, B., Vilayphiou, N., et al.: Cortical and trabecular bone microstructure did not recover at weightbearing skeletal sites and progressively deteriorated at nonweight-bearing sites during the year following international space station missions. J. Bone Miner. Res. 32, 2010-2021 (2017). https://doi.org/10.1002/jbmr.3188

Vincent, A.M., Feldman, E.L.: Control of cell survival by IGF signaling pathways. Growth Horm IGF Res. 12, 193-197 (2002). https://doi.org/10.1016/S1096-6374(02)00017-5

Vorselen, D., Roos, W.H., MacKintosh, F.C., et al.: The role of the cytoskeleton in sensing changes in gravity by nonspecialized cells. FASEB J. 28, 536-547 (2014). https://doi.org/10.1096/fj.13236356

Wei, J., Li, H., Wang, S., et al.: Let-7 enhances osteogenesis and bone formation while repressing adipogenesis of human stro$\mathrm{mal} /$ mesenchymal stem cells by regulating HMGA2. Stem. Cells Dev. 23, 1452-1463 (2014). https://doi.org/10.1089/scd.2013. 0600

Winer, J.P., Janmey, P.A., McCormick, M.E., Funaki, M.: Bone marrow-derived human mesenchymal stem cells become quiescent on soft substrates but remain responsive to chemical or mechanical stimuli. Tissue Eng. Part A 15, 147-154 (2009). https://doi.org/ 10.1089/ten.tea.2007.0388

Wong, T.Y., Chen, Y.H., Liu, S.H., et al.: Differential proteomic analysis of human placenta-derived mesenchymal stem cells cultured on normal tissue culture surface and hyaluronan-coated surface. Stem Cells Int. 2016, 2809192 (2016). https://doi.org/ 10.1155/2016/2809192

Yan, M., Wang, Y., Yang, M., et al.: The effects and mechanisms of clinorotation on proliferation and differentiation in bone marrow mesenchymal stem cells. Biochem. Biophys. Res. Commun. 460, 327-332 (2015). https://doi.org/10.1016/j.bbrc.2015.03.034

Zayzafoon, M., Gathings, W.E., McDonald, J.M.: Modeled microgravity inhibits osteogenic differentiation of human mesenchymal stem cells and increases adipogenesis. Endocrinology 145, 2421-2432 (2004). https://doi.org/10.1210/en.2003-1156 